\title{
Second annual meeting of the International Society for Quality of Life Research: Abstracts of the contributed papers
}

The following abstracts from the International Society for Quality of Life Research were inadvertantly left out of Quality of Life Research Volume 4 Number5 1995: M-L Essink-Bot, P. F. M. Krabbe, G. J. Bonsel and N. K. Aaronson; V. Peto, C. Jenkinson and R. Fitzpatrick; C. Jenkinson and R. Layte; R. J. Launois, J. Reboul-Marty, B. Henry, S. Corcaud, C. Salvadori and C. Vilain; R. J. Launois, J. Reboul-Marty and B. Henry; P. R. Reese and A. Joseph.

The Publishers and the conference organisers apologise for any inconvenience caused by their ommission.

The following abstracts were submitted after the deadline specified but were accepted for poster presentation: C. E. Selai and M. R. Trimble; S. Bito and S. Fukuhara; and D. Kerr.

\section{An empirical comparison of four generic health status measures: NHP, SF-36, COOP/WONCA Charts and the EuroQoL-instrument}

\section{M-L Essink-Bot, P. F. M. Krabbe, G. J. Bonsel and N. K. Aaronson}

Department of Public Health, Erasmus University, Rotterdam, The Netherlands (M-L. Essink-Bot, P. F. M. Krabbe); Department of Clinical Epidemiology and Biostatistics, Academic Medical Centre, Amsterdam, The Netherlands (G. J. Bonsel); The Netherlands Cancer Institute, Amsterdam, The Netherlands (N. K. Aaronson)

The aim of the study was an empirical head-to-head comparison of four generic measures for health status with respect to feasibility, reliability, internal structure, construct validity and discriminatory power. The study was motivated by the fact that testing properties are population specific (for example, if an instrument performs well in a population of seriously ill cardiac patients, this may not apply to patients with low back pain) and few publications have addressed the relative performance of generic health status measuring instruments. The NHP, the SF-36, the COOP/WONCA charts and the EuroQol descriptive instrument were simultaneously employed in a controlled survey measuring the impact of migraine on health status ( $n=$ $1,011)$. The number of missing cases per item was employed as an empirical indicator for feasibility. The internal consistency of the NHP and the SF-36 multi-item scales was determined with Cronbach's $\alpha$. The intemal structure of the four instruments was examined with use of correlation techniques [intraclass correlation coefficients (ICC) and polychoric correlation coefficients (PCC), respectively]. Construct validity was examined, firstly, by examining the patterns of ICCs between the scales of the NHP and the SF-36, and of PCCs between the items of the COOP/ WONCA and the EuroQol instrument; and secondly, by employing common factor analysis with varimax rotation to examine the relationships among the elements of the four measures. The ability of the measures to discriminate between subgroups of respondents known to differ on several relevant variables (comorbidity; absence from work due to illness) was evaluated using Mann-Whitney U-tests complemented by effect size estimations.

The NHP produced the lowest missing value rate. For the two multi-item instruments, the internal consistency of the NHP scales was lower, though acceptable, than the scales of the SF-36. Analysis of ICC matrices showed NHP scales to overlap less than SF-36. Combined factor analyses with data of the four instruments together, on the scales (NHP, SF-36) and the items (COOP/WONCA and EuroQol) respectively, resulted in two-factor solutions with a physical and a mental factor, explaining approximately $50 \%$ of variance. The SF-36 had the best power to discriminate between groups. However, these results all relate to a rather healthy population. Test-retest reliability and sensitivity to change over time could not be tested due to the survey character of the study. None of the instruments performed uniformly as 'best' or 'worst' for the characteristics studied. Generalization of this conclusion to populations with more severe illness or with other types of illness is not possible without additional empirical evidence. For the time being, the choice of the most suitable instrument for generic health status assessment in a particular study still remains to be guided by special features of each instrument.

\section{The development and validation of a short measure of well being for individuals with Parkinson's disease}

\section{Peto, C. Jenkinson and R. Fitzpatrick}

Health Services Research Unit, Department of Public Health and Primary Care, University of Oxford, Radcliffe Infirmary, Oxford OX2 6HE, UK. 
The purpose of this study was to develop and validate a health status questionnaire for use in Parkinson's disease (PDQ-39). In Stage One, questionnaire items, generated from in-depth interviews with people with Parkinson's disease, were developed into a 65 item questionnaire. In Stage Two, data from a postal survey using the 65 item questionnaire were factor analyzed (varimax rotation) to produce a shorter questionnaire (PDQ-39) with 39 items and eight scales addressing different dimensions of Parkinson's disease. In Stage Three, a second postal survey, containing the SF-36 Health Survey Questionnaire (SF-36), a generic measure of health status used to assess functioning and well being in any patient group, respondents' assessments of the severity of their Parkinson's disease symptoms, and two copies of the PDQ-39 (to be completed at a 3 day interval) was conducted to assess the reliability and validity of the PDQ-39.

The PDQ-39 proved to have good internal reliability (Cronbach's $\alpha$ for each scale) and test-retest reliability (significant correlation coefficients between scores at time 1 and time 2). Construct validity was also found to be satisfactory (correlation between the PDQ-39 and relevant SF-36 scales) and significant differences in scales scores between individuals with varying levels of self-reported symptom severity (Kruskal-Wallis ANOVA test). In addition, the PDQ-39 was found to address areas salient to Parkinson's disease, not covered by the SF-36. The PDQ-39 is a valid and reliable indicator of the impact of Parkinson's disease, and will be of value in assessing the vutcumes of management regimes, and in clinical trials.

\section{Evidence for the sensitivity of the SF-36 health status measure to inequalities in health}

\section{Jenkinson and R. Layte}

Health Services Research Unit, Department of Public Health and Primary Care, University of Oxford, Radcliffe Infirmary, Oxford OX2 6HE, UK

A recent paper suggested that the SF-36 may not be appropriate for population surveys assessing health gain due to low responsiveness (sensitivity to change) of domains on the measure. An hypothesized health gain of respondents in Social Class V to that of those in Social Class I indicated only marginal improvement in self-reported health. However, using subgroup analysis we demonstrate that the SF-36 would show dramatic changes if the health of Social Class $\mathrm{V}$ could be improved to that of Social Class I. A postal survey was conducted using a questionnaire booklet, containing the SF-36 and a number of other items concerned with lifestyles and illness. A letter outlining the purpose of the study was included. The questionnaire booklet was sent to 13,042 randomly selected subjects between the ages of $17-65$. This paper is based upon the $9,332(72 \%)$ responses gained. Outcome measures were scores for the eight dimensions of the SF-36. The sensitivity of the SF-36 was tested by hypothesizing that the scores of those in the bottom quartile of the SF-36 scores in Class V could be improved to the level of the scores from the bottom quartile of SF-36 scores in Class I using the effect size statistic.

The response rate for the questionnaire booklet was $72 \%$. We provide SF-36 scores for the population at the 25 th, 50 th and 75 th percentiles. Those reporting worse health on each dimension of the SF-36 (i.e., in the lowest $25 \%$ of scores) differ dramatically between Social Class I and V. Large effect sizes were gained on all but one dimension of the SF-36 when the health of those in the bottom quartile of the SF-36 scores in Class V were hypothesized to have improved to the level of the scores from the bottom quartile of SF-36 scores in Class I.

Analysis of SF-36 data at a population level is inappropriate. Subgroup analysis is more appropriate. The data suggests that if it were possible to improve the functioning and well being of those in worst health in Class $\mathrm{V}$ to those reporting the worse health in Class I the improvement would be dramatic. The data suggests that differences between the classes detected by the SF-36 are substantial and more dramatic than might have been previously imagined.

\section{Construction and validation of a specific health related quality of life questionnaire in chronic venous insufficiency (CVIQ)}

\section{R. J. Launois, J. Reboul-Marty, B. Henry, S. Corcaud, C. Salvadori and C. Vilain}

Département de Santé Publique-Faculté de médecine de Bobigny, Université de Paris, France (R. J. Launois, J. Reboul-Marty); ARCOS, Issy Les Moulineaux, France (B. Henry); Groupe de Recherches Servier, Neuilly, France (S. Corcaud, C. Salvadori, C. Vilain)

Patients suffering from chronic venous insufficiency of lower limbs may have their quality of life seriously altered by such an illness but existing generic quality of life instruments (NHP, SF-36 or SIP) cannot completely identify their specific complaints. The Chronic Venous Insufficiency Questionnaire (CVIQ) has been developed by iterative process. First, a focus group of 20 patients revealed a number of important aspects of quality of life with venous insufficiency other than physical symptoms of discomfort; these included anxiety, fear of travelling and concerns with physical appearance. Forty items were derived and included in a preliminary questionnaire which was administered to 150 patients.

A second study involving 2,001 subjects was planned for the reduction of items. Each participating medical doctor designated five patients suffering from venous insufficiency, and five age and sex matched controls suffering from other chronic diseases. Subjects were asked to rate on a 5-point Likert scale both the intensity of the problems 
they experienced and the importance attributed to each item. Importance items obtained from patients with venous insufficiency were used in factorial analyses (PCA, PAF). These procedures insured that the patients' preferences were considered when structuring the quality of life instrument. The final version is a 20 -item self-administered questionnaire exploring four dimensions: psychological, physical, social functionings and pain. Its internal consistency was checked for each dimension (Cronbach's $\alpha>0.820$ for three out of four factors). The quality of life scores were significantly worse for those suffering from venous insufficiency despite the morbidity of the control group. As a consequence, discriminant power of the instrument was well established by such an observational study.

Reproducibility was verified in a 60 patients test-retest study. Pearson's correlation coefficients at two week intervals were superior to 0.940 for the four-dimension subscales and the global score.

At last, the questionnaire was introduced in a randomized clinical trial including 934 patients to assess the responsiveness and the convergent validity of this instrument as well as patients' quality of life. After 2 months, subscales and global score improved and showed statistically significant differences in quality of life $(p<0.0001)$ under $1000 \mathrm{mg}$ of a micronized flavonoid fraction. At the same time, convergent validity was demonstrated: Pearson's correlation coefficients between clinical score differences and quality of life score differences were moderate (from $0.199-0.564)$ but statistically different from $0(p<0.001)$. Therefore the instrument was able to detect changes.

Reliability, face, content and construct validity as well as responsiveness were established for this specific venous insufficiency quality of life questionnaire. Such results suggest that this questionnaire may be used with confidence to measure quality of life in chronic venous insufficiency clinical trials.

\section{Evaluating the socio-economics of chemotherapic agents in metastatic breast cancer : A French perspective}

\section{R. J. Launois, J. Reboul-Marty and B. Henry \\ Département de Santé Publique-Faculté de médecine de Bobigny, Université de Paris, France (R. J. Launois, J. Reboul-Marty); ARCOS, Issy Les Moulineaux, France (B. Henry)}

A Markov process model was designed to evaluate the socio-economics of a new hemi-synthetic Vinca-Alcaloid (V) and two taxoids (TA) (TB) for second-line therapy of metastatic breast cancer. The model is based upon 28 disease states associated to clinical parameters: responses (PR, $C R$, NC, PD, EPD), toxicity (minor, acutc, cumulative) and disease complications. The content of health states description was based upon the Health Utility Index (Mark II and Mark III, McMaster University). Three dimensions: vision, hearing and speech were considered as optimal and omitted. Five specific cancer complaints were added. The scenarios were validated for comprehensibility and clarity by presenting them to five oncologists and three nurses. Preferences for health states were estimated using the standard gamble and the feeling thermometer techniques in a survey involving 20 oncology nurses from five hospitals and one outpatient clinic as a proxy for patients. To sum up the results, a method similar to the Q-TWiST approach has been used combining progression and symptomatic adverse events into a therapeutic risk-benefit index. The utility values derived by the nurses were assigned to the corresponding health states according to the amount of time duration and summed to obtain the three time periods of the Q-TWiST. Transitional probabilities, based on phase II trials, were calculated using the actuarial method and a density function approach. Evaluation of costs was based on the combined view points of the Health Care System and of the patient. Non medical direct and indirect costs were excluded from the calculation. The new French accounting cost system for DRG was used as a proxy for the economic cost of hospital stays. With this system all hospital expenses are allocated to units of outputs: depreciation cost, hotel cost, medical cost. Medical and nursing cost as well as hotel cost were allocated to DRG on a per diem basis. A weighted procedure (ICR) was used for calculating the cost of laboratory tests. Consumables such as drugs and blood were calculated on usage per patient. Linkage per patient of the successive episodes of care was obtained by examining the medical records of 153 patients in five different sites. Outpatient costs were estimated on the basis of the prescriptions made for the patient at discharge from hospital. Assignment of monetary cost for ambulatory care uses the French relative value scale and retail prices. The model keeps track of the treatment cost of the health care cost associated with adverse side effects of the treatment as well as the savings that occur, due to postponed recurrences.

Assessment of cost of quality of life under treatment from the beginning of the chemotherapy until death has been carried out following this methodology. Incremental cost-utility ratios were calculated. For a typical base line, treatments (V) and (TB) are strongly dominated by treatment (TA). Broad sensitivity analysis was carried out.

\section{Quality translations-no substitution for psychometric evaluation}

\section{P. R. Reese and A. Joseph}

\section{Glaxo Wellcome}

Increasingly, appropriate translation is recognized to include forward-backward translations, review by bilinguists and reactions from the relevant patient population; however, careful translation is not a substitute for psychometric evaluation. In preparation for a cross cultural study comparing multiple instruments, the QVM, a French 
developed migraine specific instrument, was translated using three techniques: (1) two separate and independent forward translations into American English, a reconciliation meeting between translators, backward translation, followed by focus groups with patient population (US); (2) translation into UK English by a team of professionals, a small group discussion and planned field testing (UK), and (3) simple forward-backward translation into UK English with a French/English bilingual reviewer and review for use in both the UK and US (UK/US). US and UK translation procedures met high quality standards whereas the UK/US translation utilized quicker, less sophisticated techniques; none of the translation projects shared information. This process provided an unusual opportunity for insight into the translation process and raised several questions.

Was the same educational level required to complete the questionnaire across translations? For example, the US translation used wording such as 'prolonged' and 'fatigue' whereas the UK and UK/US translations used 'long' and 'tiredness' or 'felt tired'. If educational requirements differ between the US and UK translations, use in cross-cultural studies could yield different results if patients with different educational levels were recruited. This may be particularly problematic if socioeconomic status, directly associated with educational level, impacts patient perception of quality of life, patient health status or disease severity.

Did translation choices change conceptual meaning or are these cultural differences? For example, the US translation used 'food intake', the UK translation used 'eating habits' and the UK/US translation used 'choice of food'. These may imply different meanings to patients. 'Food intake' may suggest quantity, quality and ability to select from all available foods but seems to exclude timing of meals which might be suggested by 'eating habits'. Similarly, 'choice of food' may exclude the implication of quantity and timing. Expanding or contracting the scope of conceptual meaning is problematic, although the degree of impact may be a function of the number of items used in a scale.

Do different response anchors reflect cultural differences or a severity shift? For example, does the use of a response anchor of 'very painful' to indicate a 4 on a 1-5 scale for the US and UK/US but a 5 on the UK translation affect patients' responses? A shift in severity of response anchors could result in variations between countries due to translation rather than variations in quality of life.

Do formatting and question stem differences affect responses, imply a shift in conceptual meaning, affect educational requirement or simply reflect cultural preference? Variation in the style and organization of the question stem may improve respondent comfort or may affect how patients interpret the question and select a response.

Following appropriate translation procedures is critical, but not sufficient, in providing instruments for use in crosscultural studies. Although these translations enabled visual identification of issues, translations frequently mask differences. Even where differences are visible, impact can only be assessed by comparisons of psychometric evaluations for each translation. Use in cross-cultural setting and psy- chometric evaluation including item-scale correlation, comparisons of missing responses, scale correlation with disease severity markers, correlation with general or other disease specific scales, comparisons of sample characteristics, and comparisons of psychometrics of sub-populations selected for similarity are needed to address these issues.

\section{Subjective, patient-driven assessment of quality of life in epilepsy}

\section{E. Selai and M. R. Trimble}

Institute of Neurology, National Hospital, Queen Square, London, UK

The appraisal of health-related quality of life, both in terms of what is deemed relevant and the priorities or values accorded to the areas of importance, is acknowledged to be subjective, idiosyncratic with anticipated high level of inter-subject variation. It therefore follows that the goal of the QOL researcher is to attempt to capture the individual, phenomenological experience of the HRQOL which must take account of human meanings, intentions and values. However, the goal of 'objective', scientific measurement is grounded in an empirical, positivist framework.

Attempting to satisfy both of these apparently irreconcilable research demands has lead to much methodological debate and has resulted in the variety of approaches to the assessment of HRQOL.

We present a subjective, patient-driven technique for the appraisal of HRQOL based originally on the 'repertory grid technique' (RGT) and recently streamlined to facilitate routine data collection and data analysis.

These data were collected from patients awaiting epilepsy surgery (mainly temporal lobectomy) using the RGT along side the Epilepsy Surgery Inventory (ESI-55).

\section{The role of the pilot review of score distribution and factor analysis in identifying the problem of translation of quality of life questionnaire}

\section{S. Bito and S. Fukuhara}

DGIM and Health Service Research, UCLA School of Medicine (S. Bito); Clinical Epidemiology Division, The University of Tokyo, Faculty of Medicine (S. Fukuhara)

Translation of foreign version of Quality of Life (QOL) questionnaire is not a simple task, especially when crossing a large difference in linguistic and cultural background. This paper is to demonstrate that a standard translation procedure guideline (GUIDE) (e.g. forward and backward translation, etc.) is sometimes not sufficient for the quality assurance of translation and how important the pilot review of score distribution (SCORE) and factor analysis (FA) is 
to identify potentially serious problems in translation which may be missed. We report lessons we learned from International Quality of Life Assessment project (IQOLA).

According to the GUIDE of IQOLA, we completed the translation procedure (described elsewhere) and developed the first draft of the Japanese version of the MOS short form 36 (SF-36). Before we attempted a large scale study to test validity using both healthy and patients population, we performed a pilot testing on 239 people sampled from a community. Although item-scale correlation and testretest reliability were appropriate, SCORE of physical functioning (PF) and the result of varimax rotated FA showed a very strange pattern. This result necessitated a focus group interview to several of those who answered SF-36 in this pilot study and it revealed some of them took the meaning of response choice of PF 'was the activity limited'? as 'Did your doctor limit the activity'? Upon this finding, we redid the whole procedure of translation of the response choice of $\mathrm{PF}$, followed by another independent focus group interview which confirmed its linguistic and face validity. Then the revised version was assigned to the 149 people who are randomly re-extracted from the initial 239 samples. The SCORE and the result of FA of the revised version appeared more adequate and made sense.

Although anecdotal, the above findings suggest the difficulty of translation of foreign version of QOL questionnaire and importance of critical review of SCORE and FA of the pilot data for the quality assurance of the translation.

\section{Validation of the Diabetes Quality of Life Clinical Trial questionnaire using multinational data}

\section{W. Shen, R. Brunelle and J. G. Kotsanos}

\section{Eli Lilly \& Co}

The purpose of our study is to further validate the Diabetes Quality of Life Clinical Trial Questionnaire (DQLCTQ). This health-related quality of life (QOL) questionnaire was used in multinational randomized crossover trials of patients with type I and type II diabetes in Canada, France, Germany and the USA. The questionnaire contains both generic and diabetes-specific domains consisting of existing and newly created questionnaires. The preliminary version of the DQLCTQ was evaluated in a pilot validation study prior to use in the clinical trials.

This validation analysis is unique in that it was performed in the context of clinical trials, it allowed assessment of responsiveness, an often understudied measure, and it validated newly developed instruments. The validation includes assessment of reliability, validity and responsiveness to change. This abstract focuses on validation of data for patients with type I diabetes.

Of the 468 patients in this study, $44 \%$ were female, mean age was 34 years, mean duration of diabetes was 13 years, and $56 \%$ had undergraduate or postgraduate education. Baseline data were used to evaluate reliability and validity. Three months after baseline, followup QOL data were available for the analysis of responsiveness in 431 (92\%) patients. Cronbach's $\alpha$, a measure of internal consistency reliability, was 0.70 or higher (range $0.51-0.93$ ) in all of the domains except for the diabetes worry subscale of the DQOL questionnaire used in the DCCT. Using $t$-tests, discriminant validity was assessed by comparing patients' QOL by clinical variables. Significance $(p<0.05)$ was found in several domains including general health, mental health, treatment satisfaction, DQOL total, and its selected subscales. Results of analysis on convergent validity using Pearson correlation revealed that, in general, moderate $(0.3<=r<0.5)$ or strong $(r>=0.5)$ associations exist between diabetesspecific domains, while weak $(r<0.3)$ associations exist between disease-specific and generic domains. Responsiveness to change relative to pertinent clinical variables (glycosylated hemoglobin, frequency of hypoglycemia, and clinical symptoms) were analyzed by a variety of approaches including paired $t$-test, effect size with bootstrap confidence interval, Guyatt statistic, and ROC curves. Comparative health and treatment satisfaction were found to be the most responsive domains.

In general, this analysis confirms that this questionnaire is reliable and valid for this population. Such validation analysis is a valuable and necessary step before final analysis of QOL treatment differences. As QOL is increasingly being used as an outcome measure in clinical trials, these analyses provide further understanding of the relevant QOL domains. 


\section{'Tomudex' (ZD 1694), a new direct and specific thymidylate synthase inhibitor, has resource saving implications in the management of colorectal cancer}

\section{Kerr}

Clinical Research Block, Queen Elizabeth Hospital, Birmingham B15 2TH, UK

Current modulated 5-fluorouracil regimens used in the treatment of advanced colorectal cancer are associated with significant toxicity and inconvenient administration schedules. The use of these regimens has resource and cost implications for healthcare services. Less toxic or more convenient drugs will offer real advantages in the management of this common cancer. Data were collected from a randomized multinational European IIIa trial in which 222 patients received 'Tomudex' (ZD 1694) $3 \mathrm{mg} / \mathrm{m}^{2}$ IV as a single 15 minute infusion every 3 weeks and 212 patients received 5 -fluorouracil $425 \mathrm{mg} / \mathrm{m}^{2}$ plus leucovorin $20 \mathrm{mg} / \mathrm{m}^{2}$ IV daily for five days every 4-5 weeks (5-FU-LV, Mayo regimen) until evidence of disease progression or toxicity. Patients were evaluated weekly for toxicity and 12 weekly for objective response.

At entry, the two groups were comparable in terms of their previous cancer treatment history, performance status and sites of disease. 'Tomudex' patients were 1.7 times more likely ( $p=0.059$, odds ratio 1.7$)$ to achieve complete or partial objective response to treatment (Kerr et al., BJC 1995; 7(Suppl xxv). Other indicators of palliative benefit in favour of 'Tomudex' treatment included an increased likelihood of improvement in performance status (34\% vs. $25 \%$ ) and a greater likelihood of increasing body weight by $5 \%$ or more $(15 \%$ vs. $12 \%)$. 'Tomudex' patients had a statistically significantly lower incidence $(p<0.001)$ of grade $3 / 4$ leucopenia ( $10 \%$ vs. $26 \%$ ) and mucositis ( $2 \%$ vs. $22 \%$ ). More rises in transaminases were associated with 'Tomudex', but these were usually asymptomatic and reversible. QOL was assessed using the EORTC QLQ C30 and showed similar improvement from baseline for both groups for emotional function, sleep disturbance, pain and global QOL. In addition to these tolerability and efficacy benefits, other advantages may be realized for the patient and for health care providers.

The mean length of hospital stay per cycle for drug administration was 0.7 days (mode 0.5 days) for 'Tomudex' patients compared with a mean of 3.1 days (mode 2.5 days) for patients receiving 5-FU-LV. The reduced number of dosing visits associated with 'Tomudex' administration is likely to benefit patients by decreasing the amount of travel time, often troublesome in symptomatic patients, and time away from work for patients and their carers. Single outpatient administration of a drug will also save hospital resource in terms of nursing, pharmacy and medical time creating cost saving opportunities.

Seventy-four per cent of 'Tomudex' patients were able to receive their treatment on time without significant dose reduction compared with $52 \%$ of patients receiving the Mayo regimen, as exemplified by the fact the $5.9 \%$ of 'Tomudex' patients had grade 3 or 4 haematological/nonhaematological toxicity at cycle one compared with $36.3 \%$ of 5-FU-LV patients $(p<0.001)$. In general, the fewer the dose reductions in an active cytotoxic drug regimen, the more effective the treatment is likely to be.

'Tomudex' patients required fewer days in total for treatment in ITU (9 vs. 29) and fewer community visits (69 vs. 230) than patients treated with 5-FU-LV. The frequency of outpatient visits was similar in both 'Tomudex' and 5-FULV groups (88 vs. 95), however, 'Tomudex' patients required more days on the ward (1048 vs. 789 ) (although these data are difficult to interpret due to protocol driven requirements and the investigational nature of 'Tomudex').

'Tomudex' is at least as clinically active as 5-FU-LV in the treatment of advanced colorectal cancer, but is better tolerated with a reduction in serious side effects. The simple and convenient administration regimen of 'Tomudex' reduces the associated hospital/nursing time for reconstitution and administration which coupled with a reduction in the number of dosing visits per cycle and improved ease of patient management all suggest that associated costs in these areas would be reduced and that a fuller cost analysis should be undertaken.

'Tomudex' is a trade mark, the property of Zeneca Limited.
Figure 1. Ease of management

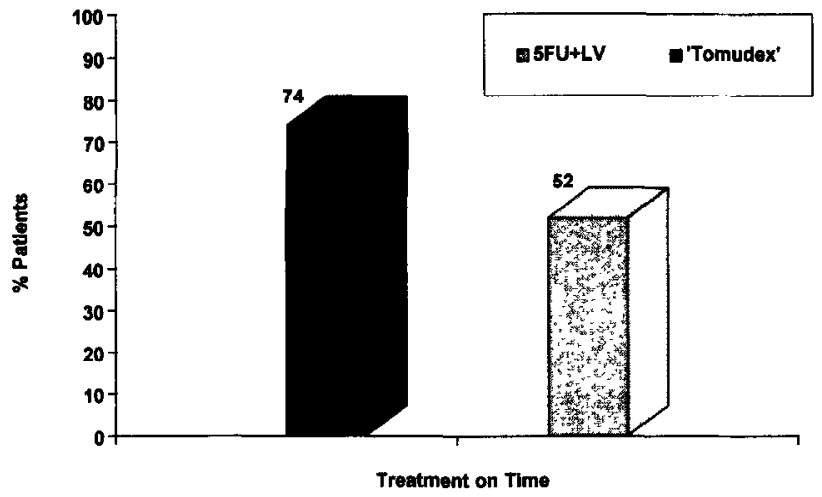

Figure 2. Influence of cycle

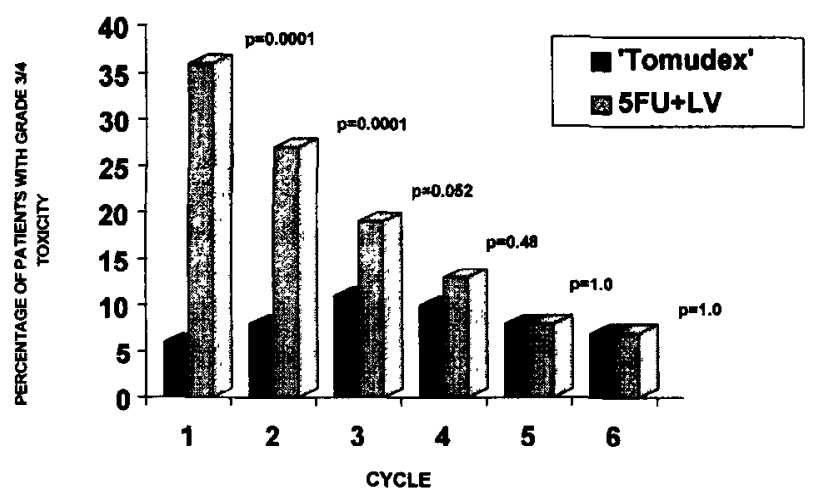

